\title{
Clinicopathological study of surgery for pulmonary metastases of hepatoblastoma with indocyanine green fluorescent imaging
}

\author{
Misa Yoshida ${ }^{1}$, Mio Tanaka ${ }^{1}$, Norihiko Kitagawa ${ }^{1}$, Masato Shinkai ${ }^{1}$, Hiroaki Goto ${ }^{1}$, and \\ Yukichi Tanaka ${ }^{1}$ \\ ${ }^{1}$ Kanagawa Childrens Medical Center
}

February 8, 2021

\begin{abstract}
Background: The prognosis of metastatic hepatoblastoma remains poor; to improve it, pulmonary metastasis must be controlled. Indocyanine green (ICG) fluorescent imaging has been used recently for lung metastasectomy. The objective of our study was to clarify the usefulness of ICG imaging for lung metastasectomy of hepatoblastoma using detailed clinicopathological analysis. Procedure: Patients with hepatoblastoma who underwent resection of pulmonary metastases with ICG fluorescent imaging were studied using retrospective analysis of clinical information, a review of their surgical records, and a histological analysis of their metastatic nodules. Results: Sixteen patients were enrolled. In total, 61 ICG-imaging-guided pulmonary metastasectomies were performed, and 350 ICG-positive and 23 ICG-negative specimens were identified. Tumors were confirmed in 250 of the ICG-positive specimens, including eight nonpalpable nodules, on microscopic examination. One hundred ICGpositive specimens and histologically tumor-negative specimens showed histological changes suggesting the regression of a tumor or bloodstream disturbance. The palpable ICG-negative tumors showed more-severe atypia than the ICG-positive tumors. Conclusions: This study demonstrates the high sensitivity of ICG imaging in detecting metastatic lesions of hepatoblastoma. Histological examinations suggested that ICG imaging detects not only tumor cells, but also nontumorous pulmonary tissues affected by bloodstream disturbance. Because a number of false-positive specimens were detected, further optimization of the dose of ICG and the timing of its administration may be required for thorough metastasectomy. Several false-negative specimens were also detected, suggesting the presence of ICG-negative metastatic tumors. Palpation during operation and imaging studies remain essential for detecting metastatic lesions, even in the era of ICG imaging.
\end{abstract}

\section{RESULTS}

\section{Patients Characteristics and Clinical Courses}

Sixteen patients were enrolled in this study (Table 1). The age at diagnosis ranged from 4 months to 11 years. Of the 16 patients, 10 had pulmonary metastasis or metastases at the initial hospital visit. The period from initial diagnosis of hepatoblastoma to the diagnosis of pulmonary metastasis was $0-30$ months. The observation period of the patients from the initial diagnosis was 2-55 months (median, 46 months). Three patients died of the disease and thirteen are still alive.

\section{Histological Examination}

In total, 61 ICG-imaging-guided pulmonary metastasectomies were performed in these patients (1-13 operations per patient), and a total of 373 specimens were examined (Table 2).

Of these 373 specimens, 350 were ICG positive during surgery, and 23 specimens were ICG-negative (and were detected by palpation by the surgeons). Tumorous tissue was confirmed in 250 of the 350 ICG- 
positive specimens on microscopic examination, whereas no tumorous tissue was identified in the other 100 specimens. Tumorous tissue was confirmed in 20 of the 23 ICG-negative specimens, whereas the other 3 specimens contained no tumorous tissue. Therefore, the sensitivity of ICG imaging in detecting pulmonary metastases of hepatoblastoma was 0.93 , and the positive predictive value was 0.71 . Of the 250 ICG-positive and histologically tumor-positive specimens, 242 included a palpable nodule or nodules, whereas 8 had no palpable nodules.

Of the 100 ICG-positive and histologically tumor-negative specimens, 98 showed one or more histological change other than tumor. Thrombus was noted in seven specimens and osteoid or chondroid formation in eleven. These findings suggest the histological changes that occur after the regression of a metastatic tumor. Nonspecific histological changes, including fibrosis, hemorrhage, and hemosiderin pigmentation, which might not be associated with the tumor, were observed in 93 specimens (Fig. 2).

Of the 23 specimens with palpable ICG-negative tumors, 20 showed a tumorous lesion or lesions on histological examination. Most of these tumors were in patient 5 , in whom the frequency of ICG-negative tumors detected increased markedly after the third operation.

The histological subtype of the metastatic lesions was epithelial type in 15 cases (predominantly embryonal type, with some mitotically active fetal subtype), and macrotrabecular type in one patient (Fig. 3A). No specimen showed the fetal type, fetal-subtype-predominant mixed fetal or embryonal type, or mesenchymal type. The ICG-negative tumors showed more-severe atypia than the ICG-positive tumors (Fig. 3B).

\section{DISCUSSION}

This study demonstrates the high sensitivity of ICG imaging, with which tumors $<1 \mathrm{~mm}$ in diameter could be detected, although a considerable number of false-positive results were obtained. Histological examination of several ICG-positive tumor-negative specimens revealed thrombi, hemorrhage, and hemosiderin pigmentation, implying disturbances of the microenvironmental pulmonary bloodstream. These histological findings suggest that ICG is detected not only when it is absorbed by tumor cells, but also when it remains in the pulmonary tissue because the bloodstream is disturbed. This hypothesis is reasonable insofar as ICG is used for fundus angiography and lymphangiography during various operations ${ }^{13,14,16-18}$. Because ICG may be distributed unevenly when the lesions are very small, it is difficult to distinguish whether the tumors themselves are detected with ICG imaging or if it is the surrounding tissues that are affected by the disturbance of the bloodstream associated with the tumor.

The histological subtype of all true-positive specimens was the embryonal type, and no mesenchymal component was detected. Therefore, the applicability of ICG imaging to lesions composed of a predominantly mesenchymal component, which are occasionally observed after chemotherapy, has not yet been clarified.

In this study, 8 of the 250 ICG-positive lesions were not palpable during surgery, and viable tumor nodules were identified in these specimens on histological examination. These lesions may not be detectable without ICG imaging. All these lesions were small, and ICG imaging might not detect the tumors themselves, but it might usefully detect circulatory disturbances in the surrounding tissues. Consequently, ICG imaging is also useful in detecting these small lesions, which are often $<1 \mathrm{~mm}$ in diameter.

Twenty specimens were ICG negative but contained palpable nodules, and viable tumors were confirmed on histological examination. Of these 20 specimens, 19 were taken from the same patient. The specimens from this patient were initially ICG positive, but reverted to ICG negative, so the character of the tumors was considered to have changed. These tumors might have become refractory to the uptake of ICG, or might have discharged ICG very rapidly. The remaining specimen might not have taken up ICG, probably in response to a circulatory disturbance. Small nonpalpable lesions that failed to take up ICG for some reason could not be detected during surgery. 
The microscopic examination of a considerable number of ICG-positive tumor-negative (false-positive) specimens also revealed nonspecific histological changes, such as fibrosis. To identify a false-positive specimen during surgery is considered difficult. To achieve thorough metastasectomy with shorter surgeries and fewer operations, further optimization of the dose of ICG and the timing of its administration may be essential. To minimize the resected lung volume, a wedge-shape resection with a minimal margin performed with open thoracotomy may be more efficient than linear-stapler thoracoscopic surgery. ${ }^{10}$

Although the sensitivity of ICG imaging is high, several false-negative (ICG-negative tumor-positive) specimens are confirmed, suggesting that some metastatic tumors cannot be detected with ICG imaging. Recognizing the limitations of metastasectomy with ICG imaging is also necessary. Palpation during operation and preoperative imaging studies may still be mandatory for the detection of metastatic lesions, even in the era of ICG imaging.

\section{Conflicts of Interest Statement}

The authors report that they have no conflicts of interest.

\section{Source of Funding}

Financial support for this study was provided by Kanagawa Children's Medical Fund.

\section{References}

1. Aronson DC, Czauderna P, Maibach R, Perilongo G, Morland B. The treatment of hepatoblastoma: Its evolution and the current status as per the SIOPEL trials. J Indian Assoc Pediatr Surg 2014;19:201-207.

2. O’Neill AF, Towbin AJ, Krailo MD, Xia C, Gao Y, McCarville MB, Meyers RL, McGahren ED, Tiao GM, Dunn SP, Langham MR, Jr., Weldon CB, Finegold MJ, Ranganathan S, Furman WL, Malogolowkin M, Rodriguez-Galindo C, Katzenstein HM. Characterization of pulmonary metastases in children with hepatoblastoma treated on Children's Oncology Group Protocol AHEP0731 (The treatment of children with all stages of hepatoblastoma): A report from the Children's Oncology Group. J Clin Oncol 2017;35:3465-3473.

3. Spector LG, Birch J. The epidemiology of hepatoblastoma. Pediatr Blood Cancer 2012;59:776-779.

4. von Schweinitz D. Hepatoblastoma: recent developments in research and treatment. Semin Pediatr Surg 2012;21:21-30.

5. Hishiki T, Matsunaga T, Sasaki F, Yano M, Ida K, Horie H, Kondo S, Watanabe K, Oue T, Tajiri T, Kamimatsuse A, Ohnuma N, Hiyama E. Outcome of hepatoblastomas treated using the Japanese Study Group for Pediatric Liver Tumor (JPLT) protocol-2: report from the JPLT. Pediatr Surg Int 2011;27:1-8.

6. Black CT, Luck SR, Musemeche CA, Andrassy RJ. Aggressive excision of pulmonary metastases is warranted in the management of childhood hepatic tumors. J Pediatr Surg 1991;26:1082-1085; discussion 1085-1086.

7. Meyers RL, Katzenstein HM, Krailo M, McGahren ED III, Malogolowkin MH. Surgical resection of pulmonary metastatic lesions in children with hepatoblastoma. J Pediatr Surg 2007;42:2050-2056.

8. Passmore SJ, Noblett HR, Wisheart JD, Mott MG. Prolonged survival following multiple thoracotomies for metastatic hepatoblastoma. Med Pediatr Oncol 1995;24:58-60.

9. von Schweinitz D, Hecker H, Harms D, Bode U, Weinel P, Burger D, Erttmann R, Mildenberger H. Complete resection before development of drug resistance is essential for survival from advanced hepatoblastomaa report from the German Cooperative Pediatric Liver Tumor Study HB-89. J Pediatr Surg 1995;30:845-852.

10. Kitagawa N, Shinkai M, Mochizuki K, Usui H, Miyagi H, Nakamura K, Tanaka M, Tanaka Y, Kusano M, Ohtsubo S. Navigation using indocyanine green fluorescence imaging for hepatoblastoma pulmonary 
metastases surgery. Pediatr Surg Int 2015;31:407-411.

11. Hunton DB, Bollman JL, Hoffman HN. Studies of hepatic function with indocyanine green. Gastroenterology 1960;39:713-724.

12. Landsman ML, Kwant G, Mook GA, Zijlstra WG. Light-absorbing properties, stability, and spectral stabilization of indocyanine green. J Appl Physiol 1976;40:575-583.

13. Craandijk A, Van Beek CA. Indocyanine green fluorescence angiography of the choroid. Br J Ophthalmol 1976;60:377-385.

14. Kim S, Lim YT, Soltesz EG, De Grand AM, Lee J, Nakayama A, Parker JA, Mihaljevic T, Laurence RG, Dor DM, Cohn LH, Bawendi MG, Frangioni JV. Near-infrared fluorescent type II quantum dots for sentinel lymph node mapping. Nat Biotechnol 2004;22:93-97.

15. Nitori N, Deguchi T, Kubota K, Yoshida M, Kato A, Kojima M, Kadomura T, Okada A, Okamura J, Kobayashi M, Sato T, Beck Y, Kitagawa Y, Kitajima M. Successful treatment of non-occlusive mesenteric ischemia (NOMI) using the HyperEye Medical System for intraoperative visualization of the mesenteric and bowel circulation: report of a case. Surg Today 2014;44:359-362.

16. Noura S, Ohue M, Seki Y, Tanaka K, Motoori M, Kishi K, Miyashiro I, Ohigashi H, Yano M, Ishikawa O, Miyamoto Y. Feasibility of a lateral region sentinel node biopsy of lower rectal cancer guided by indocyanine green using a near-infrared camera system. Ann Surg Oncol 2010;17:144-151.

17. Ogata F, Azuma R, Kikuchi M, Koshima I, Morimoto Y. Novel lymphography using indocyanine green dye for near-infrared fluorescence labeling. Ann Plast Surg 2007;58:652-655.

18. Schaafsma BE, Verbeek FP, Rietbergen DD, van der Hiel B, van der Vorst JR, Liefers GJ, Frangioni JV, van de Velde CJ, van Leeuwen FW, Vahrmeijer AL. Clinical trial of combined radio- and fluorescence-guided sentinel lymph node biopsy in breast cancer. Br J Surg 2013;100:1037-1044.

19. Kogon B, Fernandez J, Kanter K, Kirshbom P, Vincent B, Maher K, Guzetta N. The role of intraoperative indocyanine green fluorescence angiography in pediatric cardiac surgery. Ann Thorac Surg 2009;88:632-636.

20. Aoki T, Yasuda D, Shimizu Y, Odaira M, Niiya T, Kusano T, Mitamura K, Hayashi K, Murai N, Koizumi T, Kato H, Enami Y, Miwa M, Kusano M. Image-guided liver mapping using fluorescence navigation system with indocyanine green for anatomical hepatic resection. World J Surg 2008;32:1763-1767.

21. Gotoh K, Yamada T, Ishikawa O, Takahashi H, Eguchi H, Yano M, Ohigashi H, Tomita Y, Miyamoto Y, Imaoka S. A novel image-guided surgery of hepatocellular carcinoma by indocyanine green fluorescence imaging navigation. J Surg Oncol 2009;100:75-79.

22. Ishizawa T, Fukushima N, Shibahara J, Masuda K, Tamura S, Aoki T, Hasegawa K, Beck Y, Fukayama M, Kokudo N. Real-time identification of liver cancers by using indocyanine green fluorescent imaging. Cancer 2009;115:2491-2504.

23. Satou S, Ishizawa T, Masuda K, Kaneko J, Aoki T, Sakamoto Y, Hasegawa K, Sugawara Y, Kokudo N. Indocyanine green fluorescent imaging for detecting extrahepatic metastasis of hepatocellular carcinoma. J Gastroenterol 2013;48:1136-1143.

24. Yamamichi T, Oue T, Yonekura T, Owari M, Nakahata K, Umeda S, Nara K, Ueno T, Uehara S, Usui N. Clinical application of indocyanine green (ICG) fluorescent imaging of hepatoblastoma. J Pediatr Surg 2015;50:833-836.

Figure legends

FIGURE 1 ICG fluorescent imaging during pulmonary metastasectomy. A small lesion (arrow in A) taking up ICG is visualized with the Photodynamic Eye machine (B). 
FIGURE 2 Microscopic findings of ICG-stained false-positive specimens. Thrombus (A) and wellcircumscribed fibrotic mass accompanying osteoid and chondroid metaplasia (B), suggesting histological changes after the regression of a tumor, were seen in several specimens. Hemorrhage with hemosiderin-laden histiocytes was commonly seen (C). Hematoxylin-eosin.

FIGURE 3 Microscopic findings of metastatic lesions from hepatoblastoma. A. Representative example of ICG-positive, tumor-positive specimens. Image shows embryonal type. B. Representative example of ICG-negative, tumor-positive specimens, which displayed more atypical features than ICG-positive tumors. Hematoxylin-eosin.

\section{Hosted file}

Table 1.pdf available at https://authorea.com/users/394512/articles/507886clinicopathological-study-of-surgery-for-pulmonary-metastases-of-hepatoblastoma-withindocyanine-green-fluorescent-imaging
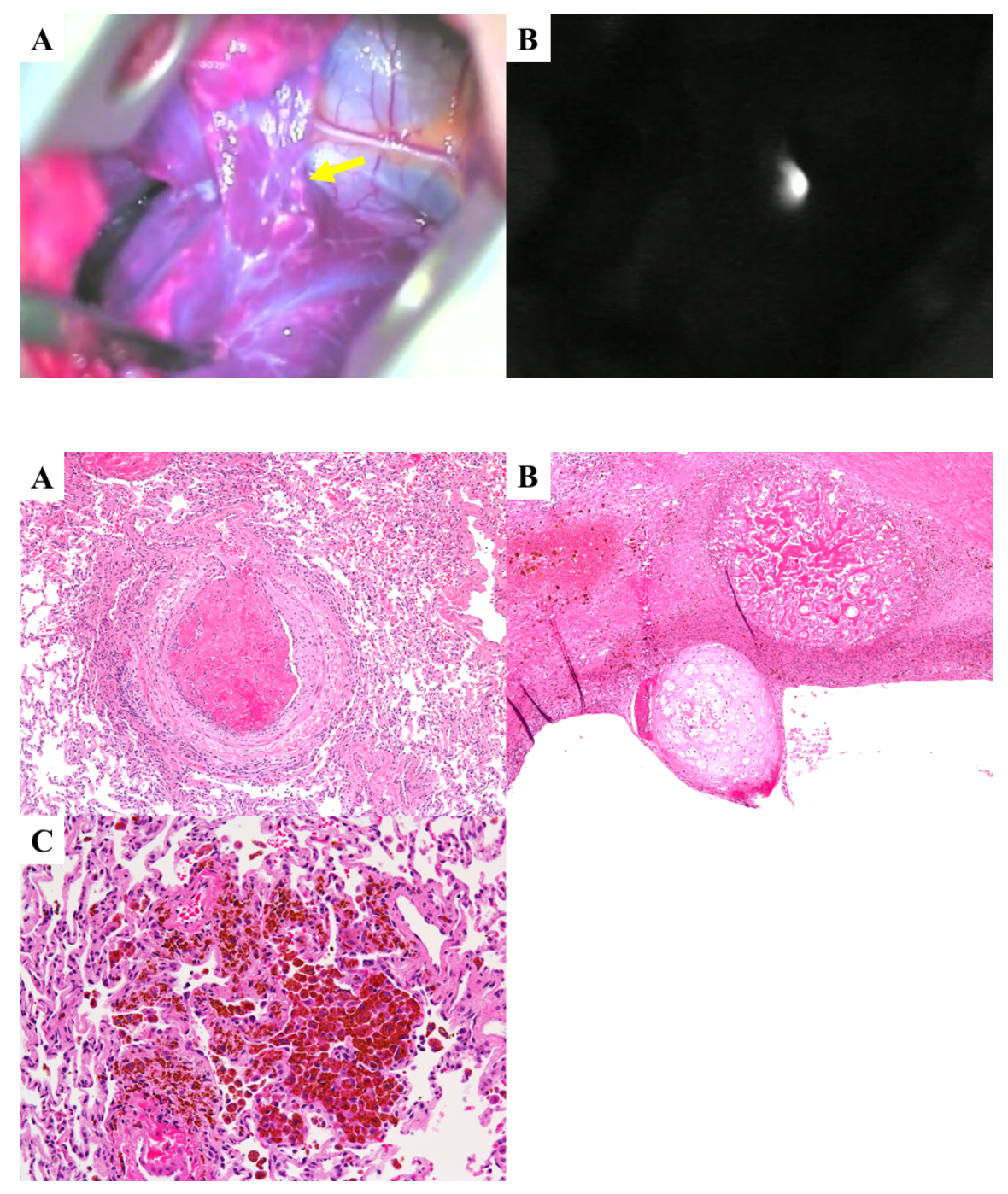


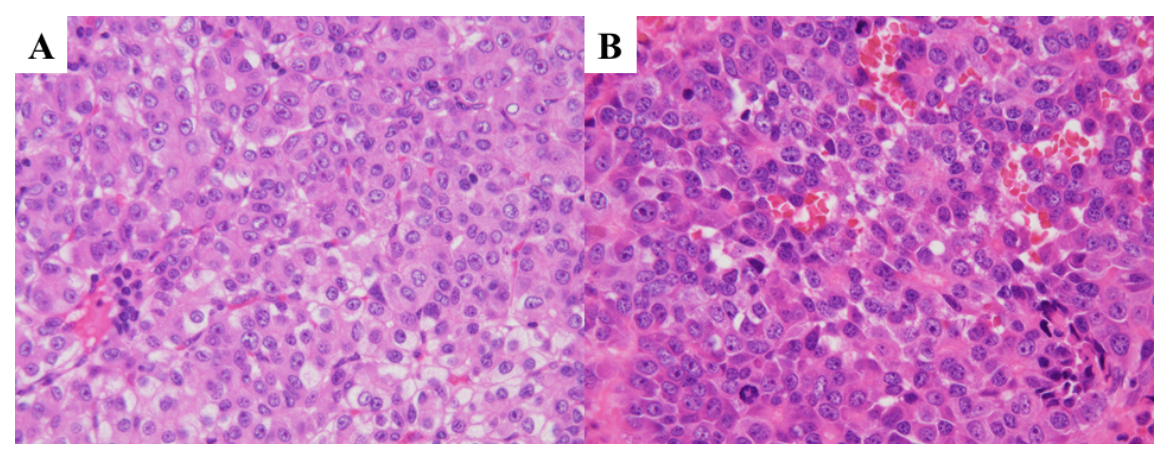

